\title{
Intermittent control of unstable multivariate systems*
}

\author{
I. Loram ${ }^{1}$, P. Gawthrop ${ }^{2}$ and H. Gollee ${ }^{3}$
}

\begin{abstract}
A sensorimotor architecture inspired from biological, vertebrate control should (i) explain the interface between high dimensional sensory analysis, low dimensional goals and high dimensional motor mechanisms and (ii) provide both stability and flexibility. Our interest concerns whether single-input-single-output intermittent control (SISO_IC) generalized to multivariable intermittent control (MIC) can meet these requirements.

We base MIC on the continuous-time observer-predictorstate-feedback architecture. MIC uses event detection. A system matched hold (SMH), using the underlying continuoustime optimal control design, generates multivariate open-loop control signals between samples of the predicted state. Combined, this serial process provides a single-channel of control with optimised sensor fusion and motor synergies. Quadratic programming provides constrained, optimised equilibrium control design to handle unphysical configurations, redundancy and provides minimum, necessary reduction of open loop instability through optimised joint impedance.

In this multivariate form, dimensionality is linked to goals rather than neuromuscular or sensory degrees of freedom. The biological and engineering rationale for intermittent rather than continuous multivariate control, is that the generalised hold sustains open loop predictive control while the open loop interval provides time within the feedback loop for online centralised, state dependent optimisation and selection.
\end{abstract}

\section{INTRODUCTION}

The vertebrate sensorimotor system conserves a common pattern across all species. The common neuroanatomy includes a spinal cord within vertebra interfacing muscles and local sensors with brain stem, cortex, and their associated basal ganglia and cerebellar loops. All vertebrates contain local sensor motor coupling within the spinal cord, and also contain basal ganglia which provide centralised mechanisms for gating, sequential selection and reinforcement learning of the coupling between sensory features and motor primitives $[2,3]$. All vertebrates contain cerebella nuclei which provide centralised mechanisms for tuning and optimising sensorimotor coupling $[4,5]$.

Vertebrates combine continuous and event related processing. Central, sequential, discrete, event related motor decision making machinery is associated with basal ganglia and frontal-striatal networks. Central decision making and optimisation confers behavioural and evolutionary advantage. The adaptive value of online decision making includes being

*Supported by EPSRC EP/F068514/1, EP/F069022/1, EP/F06974X/1 1School of Healthcare Science, Manchester Metropolitan University, Manchester, UK; email: i.loram@mmu.ac.uk

${ }^{2}$ Melbourne School of Engineering, The University of Melbourne, Melbourne, VIC, Australia; email Peter.Gawthrop@unimelb.edu.au

${ }^{3}$ School of Engineering, University of Glasgow, Glasgow, UK; email henrik.gollee@glasgow.ac.uk unpredictable for competitors, prey or predators and providing a basis for trial and error learning which requires the exploration of unknown domains.

Adaptive control must address the dual demands of regulating motor control and learning new motor behaviour. There is a tension between the dual demands of exploitation and exploration, or alternatively between stability and plasticity [6]. This tension is maximised when the controlled system is unstable, such as the multi-link mechanical structure of the upright human. An unstable mechanical structure benefits from a high control bandwidth. If regulation and stability is too good, the sensorimotor signals have low variability and bandwidth which compromises effective learning and adaptation. Vertebrate species operate successfully in a variety of environmental niches. However, if "success" depends upon adaptability and flexibility, then regulation and stability need only be minimally sufficient, while biological priority is given to a high bandwidth of decision making. Maximising the bandwidth of motor decision making requires that selection and optimisation are implemented within the sensorimotor feedback loop. A bioinspired architecture suitable for adaptable robots should reproduce that flexibility, adaptability, and low control bandwidth observed progressively through higher mammalian vertebrates. That architecture should also explain how selection and optimisation are implemented during ongoing sensorimotor control.

Within the continuous optimal feedback control paradigm discrete decision making is restricted typically to an undeclared, higher process which passes optimised control parameters to a lower continuous regulatory loop [7-9]. The continuous regulatory loop models the fast, reflexive spinal, brainstem and trans-cortical responses that have been studied extensively by physiologists $[10,11]$.

Investigation from many authors has considered whether sensorimotor control is intermittent [11-15]. The intermittent rather than continuous use of sensory information to update control signals implies an event trigger determining when to use sensory information, a discrete sampling/initialisation process and a hold process constructing a time varying control trajectory [1]. The effect of the open loop interval is to reduce the control bandwidth. The benefit is (i) the event related possibility to iteratively reinitialise the control basis and (ii) the availability of predictively stabilised open loop time to provide state dependent optimisation [11, 16-18]. In short, intermittent v. continuous control trades online flexibility for control bandwidth. Recent theoretical and methodological advances in SISO_IC have provided new experimental evidence of sequential, refractory response selection during sustained sensorimotor control [7, 11, 19, 20]. While including continuous control as a special case, SISO_IC provides a more general computational paradigm in which discrete refractory selection occurs as a serial, 


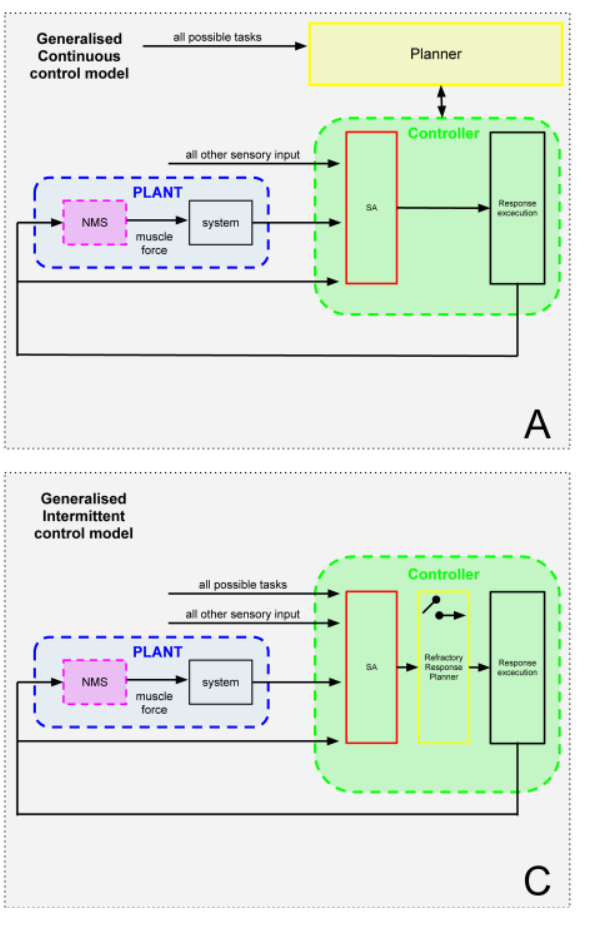

Figure 1. A Continuous optimal control: task selection occurs at the "Planner" level, the selected strategy is employed continuously via the low level feedback mechanism. This feedback loop consists of the "Controller" enclosing the continuous stages of sensory analysis (SA) and response execution (RE).

$\boldsymbol{B}$ typical implementation with observer and state FB blocks. The command signal serves as a single input to the "Plant" whose neuro-muscular system (NMS; panel E) synergistically (e.g. pattern generators, muscle modes, synergies or optimal feedback systems) translates it to the multiple muscles according to its current parameter settings. Once these are routed, the underlying muscle forces actuate the multi-segmental system in the space of the elemental variables.

C Generalised IC: a "refractory Response Planner" forms the intermediate stage between sensory analysis (SA) and response execution (RE); an online process of selecting one movement alternative from the many possible which occurs within the feedback loop that regulates the task. D MIC implementation [1].

$\boldsymbol{E}$ typical NMS block where k is the feedback gain, Sigma generates a weighted sum of muscle forces and X_ss synergistically allocates the desired forces to each muscle.

sequential, single channel process within the main feedback loop [21-23].

Here, our question is whether SISO_IC generalised to MIC can stabilise the unstable, structure of the upright human, using accepted values of passive joint stiffness.

\section{Methods}

A number of SISO models of intermittent control are available [14, 24, 25], one of which has been developed to control two segments [15]. The approaches are compared by [26]. This paper is based on the explicit SISO model [21] which has been experimentally verified $[7,19,20]$ and which has been extended, recently to multivariate form [1].

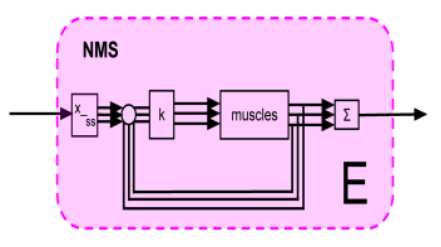

Control of multiple neuromuscular degrees of freedom. Vertebrate movement is characterised by lowdimensional goals achieved using high-dimensional muscle input. In control system terms the system has redundant actuators. One approach to redundancy is by using the concept of synergies [14] defined as groups of muscles which act in concert to give a desired action. It has been shown that synergies arise naturally in the context of optimal control [9]. Synergies may be arranged in hierarchies. For example, in the context of posture, there is a natural three-level hierarchy with increasing dimension comprising task space, joint space and muscle space. This model uses the task space - joint space hierarchy.

Fusion of multiple sensory modalities. In control system terms, the abundant vertebrate, sensor redundancy can be incorporated into state-space control using observers or Kalman-Bucy filters; this is the dual of the optimal control problem. Again sensors can be arranged in a hierarchical fashion. Hence optimal control and filtering provides the basis for a continuous-time control system that simultaneously applies sensor fusion to utilise sensor redundancy and optimal control to utilise actuator redundancy. The extension of SISO_IC [21] to MIC [1] allows conventional treatment of multiple sensors and actuators using linear quadratic design.

Extending SISO_IC, MIC is based on the continuoustime optimal control design method using the observer, predictor state-feedback architecture [1]. MIC uses an event detection mechanism. A system matched hold, using the underlying continuous optimal control design, generates multivariate open-loop control signals between samples of the predicted state. Combined, this serial, sequential process provides a single-channel of control with optimised sensor fusion and motor synergies. [1].

The system-matched hold (SMH) is the key component of the intermittent control. As described previously [21], the SMH state $\hat{x}_{h}$ evolves in a local intermittent time frame $\tau$ as

$$
\begin{aligned}
& d / d t \hat{x}_{h}(\tau)=A_{h} \hat{x}_{h}(\tau) \\
& \text { where } A_{h}=A_{c} \text { and } \hat{x}_{h}(0)=\hat{x}_{p}\left(t_{i}-t_{d}\right)
\end{aligned}
$$

where $\mathbf{A}_{c}$ is the closed-loop system matrix of the equivalent continuous system, and $\hat{x}_{p}$ is the predicted state. The hold state $\hat{x}_{h}$ replaces the predictor state $\hat{x}_{p}$ in the controller equation. Other holds (where $\mathbf{A}_{h} \sim=\mathbf{A}_{c}$ ) are possible [27]. 
Equation (1) does not explicitly depend on the number of system outputs and inputs and is thus unchanged in the multivariable context. In essence, the only differences between the multivariable intermittent controller and SISO intermittent controller are related to the underlying design method for the observer gain, controller gain and equilibrium state which are handled conventionally using linear quadratic regulator (LQR) design as per continuous control.

The intermittent controller generates an open loop control signal based on the hold state $\hat{x}_{h}$ (1). At the discrete intermittent sample times $t_{i}$, the hold state is reset to the estimated system state $\hat{x}_{w}$ generated by the observer, thus feedback occurs at the intermittent sample times $t_{i}$. The sample times are constrained to be at least $\Delta_{\min }$ apart. But, in addition to this constraint, feedback only takes place when it is needed. Such feedback is required when the open-loop hold state $\hat{x}_{h}$ differs significantly from the closed-loop observer state $\hat{x}_{w}$ indicating the presence of disturbances. There are many ways to measure such a discrepancy. Following [21], the one chosen here is to look for a quadratic function of the error $\mathbf{e}_{h p}$ exceeding a threshold $q^{2}$ :

$$
\begin{aligned}
& E=e_{h p} T(t) Q_{t} e_{h p}(t)-q_{t}^{2} \geq 0 \text { (2) } \\
& \text { where } e_{h p}(t)=\hat{x}_{h}(t)-\hat{x}_{w}(t) \\
& \text { where } Q_{t} \text { is a positive semi-definite matrix. }
\end{aligned}
$$

The unstable, mechanical system comprises a linearized, sagittal (2D) version of the three link (lower leg, upper leg, trunk-arms-head), three joint (ankle, knee, hip) model of human posture given by [28]. The upper, middle and lower links are indicated by subscripts $\mathrm{u}, \mathrm{m}$ and 1 respectively. The linearized equations correspond to:

$$
M \theta *-G \theta=N T
$$

where $\theta$ is the vector of link angles, $T$ is the vector of joint torques and $M, G$, and $N$ are respectively the mass, gravity, link matrices. The values for the link lengths, CoM location, masses and moments of inertia (about CoM) where taken from Figure 4.1 and Table 4.1 of [29].

The single joint inverted pendulum model of human balance uses the effect of passive ankle joint stiffness to counteract gravity and reduce the toppling rate of the pendulum [30, 31]. This toppling rate is directly related to the maximum real part of the system eigenvalues. This is important as it reduces the control bandwidth necessary to stabilise the unstable inverted pendulum system. The situation is more complicated in the multiple link case as, unlike the single inverted pendulum case, the joint angles are distinct from the link angles. The gravity matrix is diagonal in link space. The corresponding stiffness matrix $K_{\varphi}$ is diagonal in joint space and therefore cannot cancel the gravity matrix in all configurations. The diagonal vector of mechanical joint stiffness $k_{\varphi}$ defines the stiffness for a joint when the other joints are set at zero (straight) angle

The choice of $K_{\varphi}$ directly affects mechanical instability of the multi-link system via the maximum real part of the system eigenvalues and thus influences the required closedloop control bandwidth.
By comparison with continuous control, intermittent control has a reduced control bandwidth and is complemented by the biological availability of joint stiffness arising from passive, elastic structures or co-activation of muscles. To estimate the minimal joint stiffness required we calculate minimal stiffness consistent with the required maximum real part of the system eigenvalues [1]. Minimising the mean square joint stiffness subject to a maximum open loop system real eigenvalue $\sigma_{\max }$ is a quadratic optimisation with non-linear constraints which is solved by sequential quadratic programming (SQP).

\section{RESULTS}
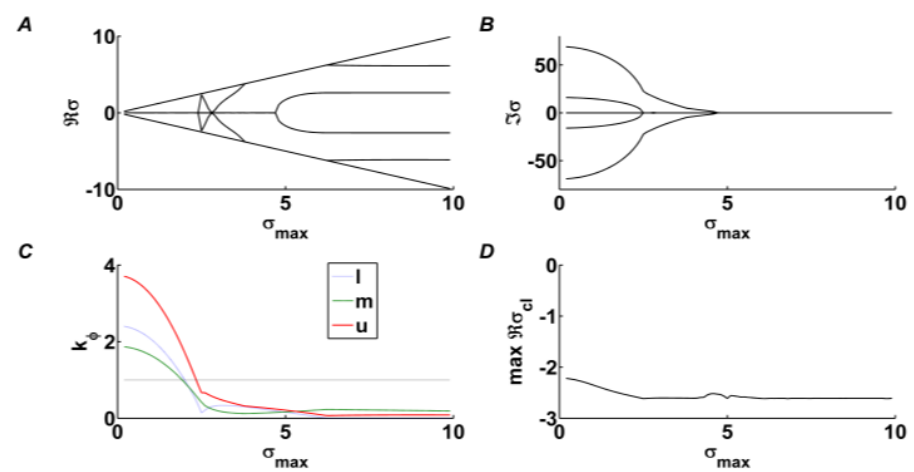

D

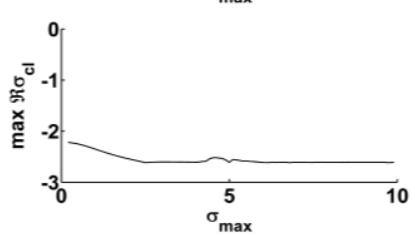

Figure $2 \boldsymbol{A}-\boldsymbol{C}$ shows respectively how the real and imaginary parts of the three system eigenvalues pairs vary with the constraint $\sigma_{\max }$ together with the spring constants $k_{\varphi}$. The joint stiffness values are normalised to the values required to cancel the effect of gravity when the other joint angles are set to zero.

$\boldsymbol{D}$ shows variation of the maximum real closed loop eigenvalue for a control design with intermittent control parameters minimum open loop interval $\left(\Delta_{\min }\right) 0.25 \mathrm{~s}$, time delay $0.1 \mathrm{~s}$, event threshold $\left(q_{t}\right)$ $0.1^{\circ}$, and optimized parameters for control priority $\left(q_{v}, q_{p}, q_{T}\right.$ for velocity position, torque).

The spring constants and imaginary parts rise rapidly when the maximum real eigenvalue is reduced to below about 2.3. When the maximum real eigenvalue lies above 3 , the joint stiffness values lies in the range $0.05-0.35$ which is at the lower end of accepted physiological estimates of the intrinsic passive joint stiffness ranging 0.2-0.9 [30-32].

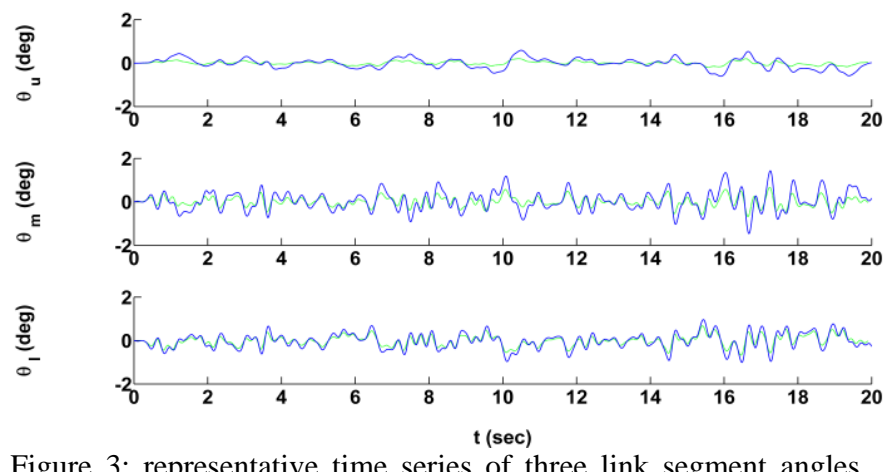

Figure 3: representative time series of three link segment angles using a white noise disturbance (rms amplitude $1 \mathrm{Nm}$, bandwidth 0.1-5 HZ, $\sigma_{\max }=3, q_{v}, q_{p}, q_{T}=0.07,5,100$ [1]) applied at the ankle joint. Using otherwise identical parameters, intermittent regulation (blue) is poorer than continuous regulation (green), but not by much. 
For a SMH using an underlying continuous LQR control design with predictor to remove the time delay from the feedback loop, the closed loop system is stable.

\section{CONCLUSIONS}

- MIC can stabilise an unstable multi-link mechanical system representing the upright adult human.

- Stabilisation is consistent with low, physiological values of intrinsic joint stiffness.

IC operates a sequential, refractory single channel process and links psychological refractoriness, normally associated with discrete serial reaction time tasks, with sustained sensorimotor control. The open loop interval explains refractoriness and explains the low bandwidth of voluntary, vertebrate control. While concurrent, parallel MICs (multitasking) are possible, combination of multiple goals into an optimised single dimensional goal implies convergence to a single, single channel process. Discrete decision making within the feedback loop, provides a mechanism for flexible artificial control consistent with the vertebrate architecture of control via centralised selection. The biological and engineering rationale for intermittent rather than continuous multivariate control, is that the generalised hold sustains open loop predictive control while the open loop interval provides time within the feedback loop for online centralised, state dependent optimisation and selection.

\section{REFERENCES}

[1] P. Gawthrop, H. Gollee, and I. Loram, "Intermittent Control in Man and Machine," in Event-based control and signal processing, ed: CRC Press/Taylor \& Francis, 2015 (Nov), p. http://arxiv.org/abs/1407.3543.

[2] P. Redgrave, "Basal ganglia," J Scholarpedia, vol. 2, p. 1825, 2007.

[3] M. J. Frank, "Computational models of motivated action selection in corticostriatal circuits," Current Opinion in Neurobiology, vol. 21, pp. 381-386, Jun 2011.

[4] K. Doya, "Complementary roles of basal ganglia and cerebellum in learning and motor control," Curr Opin Neurobiol, vol. 10, p. $732,2000$.

[5] J. C. Houk, C. Bastianen, D. Fansler, A. Fishbach, D. Fraser, P. J. Reber, et al., "Action selection and refinement in subcortical loops through basal ganglia and cerebellum," Philosophical Transactions of the Royal Society B-Biological Sciences, vol. 362, pp. 1573-1583, Sep 292007.

[6] G. A. Carpenter and S. Grossberg, "The ART of adaptive pattern recognition by a self-organizing neural network," Computer, vol. 21, pp. 77-88, 1988.

[7] C. Van De Kamp, P. Gawthrop, H. Gollee, M. Lakie, and I. D. Loram, "Interfacing sensory input with motor output: does the control architecture converge to a serial process along a single channel?," Frontiers in Computational Neuroscience, vol. 7, 2013-May-9 2013.

[8] P. Cisek, "Neural representations of motor plans, desired trajectories, and controlled objects," Cognitive Processing, vol. 6, pp. 15-24, 2005/03/01 2005.

[9] E. Todorov, "Optimality principles in sensorimotor control," Nat Neurosci, vol. 7, pp. 907-15, Sep 2004.

[10] S. H. Scott, T. Cluff, C. R. Lowrey, and T. Takei, "Feedback control during voluntary motor actions," Curr Opin Neurobiol, vol. 33, pp. 85-94, Mar 272015.

[11] I. D. Loram, C. van de Kamp, M. Lakie, H. Gollee, and P. J. Gawthrop, "Does the motor system need intermittent control?," Exerc Sport Sci Rev, vol. 42, pp. 117-25, Jul 2014.
[12] K. J. W. Craik, "Theory of the human operator in control systems. I. The operator as an engineering system," British Journal of Psychology, vol. xxxviii, p. 56, 1947.

[13] R. C. Miall, D. J. Weir, and J. F. Stein, "Manual tracking of visual targets by trained monkeys," Behavioural Brain Research, vol. 20, pp. 185-201, 5// 1986.

[14] P. D. Neilson and M. D. Neilson, "An overview of adaptive model theory: solving the problems of redundancy, resources, and nonlinear interactions in human movement control," $J$ Neural Eng, vol. 2, pp. S279-312, Sep 2005.

[15] Y. Suzuki, T. Nomura, M. Casadio, and P. Morasso, "Intermittent control with ankle, hip, and mixed strategies during quiet standing: A theoretical proposal based on a double inverted pendulum model," Journal of Theoretical Biology, vol. 310, pp. 55-79, Oct 72012.

[16] E. Ronco, T. Arsan, and P. J. Gawthrop, "Open-loop intermittent feedback control: practical continuous-time GPC," Control Theory and Applications, IEE Proceedings -, vol. 146, pp. 426434, 1999.

[17] P. Gawthrop, D. Wagg, S. Neild, and L. Wang, "Powerconstrained intermittent control," International Journal of Control, vol. 86, pp. 396-409, Mar 12013.

[18] P. J. Gawthrop and L. Wang, "Intermittent redesign of continuous controllers," International Journal of Control, vol. 83, pp. 1581-1594, 20102010.

[19] C. van de Kamp, P. J. Gawthrop, H. Gollee, and I. D. Loram, "Refractoriness in sustained visuo-manual control: is the refractory duration intrinsic or does it depend on external system properties?," PLoS Comput Biol, vol. 9, p. e1002843, 2013.

[20] I. D. Loram, C. van de Kamp, H. Gollee, and P. J. Gawthrop, "Identification of intermittent control in man and machine," $J R$ Soc Interface, vol. 9, pp. 2070-84, Sep 72012.

[21] P. Gawthrop, I. Loram, M. Lakie, and H. Gollee, "Intermittent control: a computational theory of human control," Biol Cybern, vol. 104, pp. 31-51, Feb 2011

[22] P. Gawthrop and L. Wang, "The system-matched hold and the intermittent control separation principle," International Journal of Control, vol. 84, pp. 1965-1974, 20112011.

[23] P. J. Gawthrop and L. Wang, "Event-driven intermittent control," International Journal of Control, vol. 82, pp. 22352248, 20092009.

[24] Y. Asai, Y. Tasaka, K. Nomura, T. Nomura, M. Casadio, and P. Morasso, "A Model of Postural Control in Quiet Standing: Robust Compensation of Delay-Induced Instability Using Intermittent Activation of Feedback Control," Plos One, vol. 4, Jul 82009.

[25] T. Insperger, L. L. Kovacs, P. Galambos, and G. Stepan, "Actand-Wait Control Concept for a Force Control Process with Delayed Feedback," Motion And Vibration Control, pp. 133-142, 2009.

[26] P. Gawthrop, I. Loram, H. Gollee, and M. Lakie, "Intermittent control models of human standing: similarities and differences," Biol Cybern, vol. 108, pp. 159-68, Apr 2014.

[27] P. J. Gawthrop and H. Gollee, "Intermittent tapping control," Proceedings of the Institution of Mechanical Engineers Part IJournal of Systems and Control Engineering, vol. 226, pp. 12621273, Oct 2012.

[28] A. V. Alexandrov, A. A. Frolov, F. B. Horak, P. Carlson-Kuhta and S. Park, "Feedback equilibrium control during human standing," Biological Cybernetics, vol. 93, p. 309, 2005.

[29] D. A. Winter, Biomechanics and Motor Control of Human Movement: John Wiley \& Sons, Inc., 2009.

[30] I. D. Loram, C. N. Maganaris, and M. Lakie, "The passive, human calf muscles in relation to standing: the non-linear decrease from short range to long range stiffness," J Physiol, vol. 584, pp. 661-75, Oct 152007.

[31] M. Casadio, P. G. Morasso, and V. Sanguineti, "Direct measurement of ankle stiffness during quiet standing: implications for control modelling and clinical application," Gait \& Posture, vol. 21, p. 410, 2005.

[32] T. Kiemel, A. J. Elahi, and J. J. Jeka, "Identification of the plant for upright stance in humans: multiple movement patterns from a single neural strategy," J Neurophysiol, vol. 100, pp. 3394-406, Dec 2008 